\title{
Transforming Health Facilities into Mother-Baby Friendly Centers: Experience of World Vision, East African Maternal Newborn and Child Health Project in Kitgum District, Uganda, 2016
}

\author{
Babughirana Geoffrey¹, Musasizi Benon1, Lorna Muhirwe Barungi1, Andrew Tumuhameho1, \\ Tushemerirwe Florence ${ }^{2}$, Twaha Rwegyema ${ }^{3}$ \\ ${ }^{1}$ World Vision, Kampala, Uganda \\ ${ }^{2}$ Independent Consultant, Kampala, Uganda \\ ${ }^{3}$ The Diet Clinic, Kampala, Uganda \\ Email: gbabughirana@gmail.com, musasizi.benon1@gmail.com, lornamuhirwe@gmail.com, atumuhameho@gmail.com, \\ ftushemerirwe@musph.ac.ug, twahregy@gmail.com
}

How to cite this paper: Geoffrey, B., Benon, M., Barungi, L.M., Tumuhameho, A., Florence, T. and Rwegyema, T. (2016) Transforming Health Facilities into Mother-Baby Friendly Centers: Experience of World Vision, East African Maternal Newborn and Child Health Project in Kitgum District, Uganda, 2016. Open Access Library Journal, 3: e3180.

http://dx.doi.org/10.4236/oalib.1103180

Received: October 28, 2016

Accepted: December 23, 2016

Published: December 26, 2016

Copyright $\odot 2016$ by authors and Open Access Library Inc.

This work is licensed under the Creative Commons Attribution International License (CC BY 4.0).

http://creativecommons.org/licenses/by/4.0/

\section{Abstract}

The Baby Friendly Health facility Initiative (BFHI) is a health care environment which supports, protects and promotes breastfeeding as a norm. The World Vision Uganda East African Maternal Newborn and Child Health (EAMNCH) project supported its implementation with a focus on the Transformation of 24 health facilities into Mother-Baby Friendly in Kitgum district. Four (4) out of twelve (12) health facilities that participated in the external assessment scored $75 \%$ and above, and this is the required minimum score for a health facility to be certified as Baby Friendly. The four are: St Joseph's Hospital (81\%), Orom HCiii (75\%), Loborom HCiii (75\%), and Akilok HCiii (75\%). From the findings, it's recommended that health facilities should actively engage the Village Health Teams (VHTs) in their catchment areas to strengthen Infant and Young Child Feeding (IYCF) community support to strengthen community-health facility linkages.

\section{Subject Areas}

Public Health

\section{Keywords}

Baby Friendly Health Facility Initiative, Health Worker Mentorship and Coaching, Transformation of Health Facilities, Health System's Strengthening 


\section{Introduction}

\subsection{The Global Strategy on the Baby-Friendly Hospital Initiative}

The internationally defined term "Baby-Friendly" may be used only by maternity services that have passed external assessment according to the Global Criteria for the Baby-friendly Hospital Initiative BFHI [1]. The term "Baby-Friendly" is not appropriately applied to other medical services, community activities, workplaces or commercial products. The BFHI was launched by WHO and UNICEF in 1991, following the Innocenti Declaration of 1990. The initiative is a global effort to implement practices that protect, promote and support breastfeeding. The global BFHI instruments have been expanded for integrated care. The materials reflect new research and experience, reinforce the International Code of Marketing of Breast-milk Substitutes, support mothers who are not breastfeeding, provide modules on HIV and infant feeding and motherfriendly care, and give more guidance for monitoring and reassessment.

According to the Innocenti Declaration [2], breastfeeding is a unique process that provides ideal nutrition for infants and contributes to their healthy growth and development, reduces incidence and severity of infectious diseases, thereby lowering infant morbidity and mortality, contributes to women's health by reducing the risk of breast and ovarian cancer, and by increasing the spacing between pregnancies, provides social and economic benefits to the family and the nation and provides most women with a sense of satisfaction when successfully carried out and therefore needs to be Protection, Promotion and Support through national regulation and re-enforcement.

To help in the implementation of the initiative, different tools and materials were developed, field-tested and provided, including a course for maternity staff, a selfappraisal tool, and an external assessment tool. Additional tools were developed afterwards, such as monitoring and reassessment tools. Since its launching BFHI has grown, with more than 20,000 designated facilities in 152 countries around the world. The initiative has measurable and proven impact, increasing the likelihood of babies being exclusively breastfed for the first six months [1].

To achieve BFHI accreditation, health facilities must demonstrate a rate of at least $75 \%$ exclusive breastfeeding among mothers at discharge, adherence to the International Code of Marketing Breast-milk Substitutes and successful implementation of the ten steps to successful breastfeeding, as defined by the joint WHO/UNICEF statement [3]. The accreditation process begins with having a written breastfeeding policy that is routinely communicated to all health care staff, training all health care staff in skills necessary to implement this policy, fostering the establishment of breastfeeding support groups, and referring mothers to them on discharge from the hospital or clinic.

The sustainability and scaling up of BFHI requires the integration of breastfeeding-related subjects into the curricula of health workers at all levels and expanding BFHI beyond maternity services into neonatal, pediatric, and the community health services will help contribute to an improved continuity of care and breastfeeding outcomes. 


\subsection{The Uganda BFHI Instruments}

In Uganda the initiative was launched in 1992 spear headed by Uganda Lactation Management Education Team (ULMET) [4]. In 1995 Mbale Health facility was designated baby friendly, several Health facilities over years have been declared baby friendly with support from UNICEF, WHO and IBFAN Africa. Of the 150 children who die every day in Uganda, 75 percent die due to underlying malnutrition attributed to poor infant feeding practices [5]. In addition, many children suffer long-term effects from poor infant feeding practices including impaired development, malnutrition, and increased infectious and chronic illness. The policy guidelines on Infant and Young Child Feeding [5] aim to provide the framework for ensuring the survival, and enhancing the nutrition, health, growth and development of infants and young children, as well as strengthening the care and support services to their parents and caretakers to help them achieve optimal Infant and Young Child Feeding (IYCF).

Uganda adopted the Global 10 steps to BFHI and came up with 6 additional steps (Table 1). The aim of BFHI in Uganda is to implement the Sixteen Steps to Successful Breastfeeding (IYCF) and to regulate the distribution of free and low-cost supplies of breast-milk substitutes to health facilities and mothers. BFHI provides a frame work for enabling mothers to acquire the skills they need to breastfeed exclusively for six months

Table 1 . The $16 \mathrm{BFHI}$ requirements for a health facility to be designated baby friendly.

Every facility providing maternity services and care for newborn infants should:

Step 1 . Have a written breastfeeding policy that is routinely communicated to all health care staff.

Step 2. Train all health care staff in skills necessary to implement this policy.

Step 3. Inform all pregnant women about the benefits and management of breastfeeding.

Step 4. Help mothers initiate breastfeeding within an hour of birth.

Step 5. Show mothers how to breastfeed, and how to maintain lactation even if they are separated from their infants.

Step 6. Give newborn infants no food or drink other than breast milk, unless medically indicated.

Step 7. Practice rooming-in-allow mothers and infants to remain together- 24 hours a day.

Step 8. Encourage breastfeeding on demand.

Step 9. Give no artificial teats/pacifiers (also called dummies or soothers) to breastfeeding infants.

Step 10. Foster the establishment of breastfeeding support groups and refer mothers to them on discharge from the hospital or clinic.

Step 11. Comply with the Food Safety (Marketing of Infant and Young Child Foods) Regulations Code.

Step 12. Provide Mother-friendly Care.

Step 13. Support Infant Feeding in the Context of HIV.

Step 14. Ensure that all new born babies delivered in Health Facilities or clinics receive step BCG and Polio "O" vaccine before discharge.

Step 15. Ensure that all mothers who deliver in Health Facilities or clinics receive 200,000 IU of Vitamin A Capsule before discharge.

Step 16. Issue a correctly filled in Child Health Card for each new born to the mother before discharge from the maternity ward. Provide Mother-friendly Care. 
and continue breastfeeding with the addition of complementary foods for 2 years or beyond and a Baby Friendly Health facility also assists mothers who are not breastfeeding to make informed decisions and to care for their babies as well as possible [6].

BFHI in context HIV/AIDS for Uganda [5] aims to promote support for exclusive breastfeeding and BFHI continue to be important everywhere, even in areas of high HIV prevalence. The special needs of HIV-positive women can be fully accommodated without compromising Baby Friendly Health facility status and mothers have a right to information and support that enables them make fully informed decisions about infant feeding, health and development. In addition, it is important to continue to support breastfeeding for women who are HIV negative or of unknown HIV status but if the emphasis is only on the risks of mother to child transmission of HIV through breastfeeding it may be forgotten that breastfeeding remains the best choice for most mothers and babies.

\subsection{The BFHI Situation for Kitgum District at the Inception of the Project}

BFHI implementation in Kitgum district was facilitate by the World Vision Uganda under the East African Maternal and Child Health (EAMNCH) Project. The Project utilised a Systems strengthening approach which began with orientation of the District leadership and District Health Team (DHT) on the BFHI strategy to create awareness and ownership of the intervention at district level. The baseline assessment [7] done to establish the capacity of the facilities to implement BFHI highlighted the gaps that were crucial for improved $\mathrm{MNCH}$ service delivery.

Generally, the Health facilities did not perform well. The best performing health facility (Omiyanyima Health centre iii) scored 68\% while the worst performing (Kitgum Town council Health Centre iii) scored $36 \%$. The Health facilities performed fairly well on requirement $11,12,15$ and 16 while the rest of the requirements had major challenges. These findings therefore indicated that none of the health facilities in Kitgum district were complaint or committed to BFHI based on the Ministry of Health standards. The health facilities therefore required to be supported vigorously starting with the capacity to health workers and improving the health facility system to support implementation.

The results further indicated that only $43.2 \%$ of health workers in the health units had been trained in the IYCF course. Worse still, only $2.3 \%$ of health workers had been trained in BFHI. Kitgum hospital, Omiyanyima HC III and Oryang HC II had the highest number of health workers trained in IYCF $(6.8 \%)$, followed by Kitgum matidi HC III that had $2 \%$ health workers.

$54.2 \%$ of the health workers knew that their health facilities implemented BFHI. This indicates that most health workers do not know about the BFHI approach and what it is about. $66.7 \%$ of the health workers who noted that their facilities implemented BFHI had a policy that mainly promotes exclusively breastfeeding $16.7 \%$ reported not to have a policy while the others did not know anything about the policy. This is still showed 
the level of knowledge on BFHI amongst the health workers in the district.

\subsection{Objectives of the BFHI Implementation}

The purpose of BFHI implementation in Kitgum was to contribute to the overall objective of EAMNCH project which was to contribute to improved health and nutrition status of children under 5 years, pregnant and breast feeding mothers in the district through supporting health facilities to provide "mother-baby friendly" services. Successful implementation of BFHI in health facilities evidenced by expansion of service delivery to mothers and children hence reduction in maternal and child mortality as their health is improved. The project therefore set out to:

1) To facilitate the 24 health facilities in the implementation of the sixteen steps put in place by $\mathrm{MoH}$ for BFHI.

2) To provide necessary information and equipment for the implementation of the BFHI initiative through training of health workers since they are the ones responsible for its implementation.

3) Facilitate the setup of a time bound program and a routine plan for the implementation of BFHI so as to increase its efficient and effective implementation in the district.

4) To facilitate the health facilities that have signed commitment for designation and ensure they are declared as "baby friendly" i.e. they should be offered certificates by $\mathrm{MoH}$.

\section{Methodology}

\subsection{Study Area}

The BFHI implementation was done in twenty four (24) health facilities in Kitgum district. Kitgum district is in the northern part of Uganda. It is located between Longitude $320 \mathrm{E}$, and $340 \mathrm{E}$, Latitude $020 \mathrm{~N}$ and $040 \mathrm{~N}$. It is bordered by Lamwo District in the North, the Republic of South Sudan in the North East, Kotido District in the East, Agago District in South East, Pader District in the South and Gulu District in the Northwest. Based on the 2014 population Census, Kitgum District has an estimated population of 204,012 of which 105,574 are females while 98,438 are males. The district average Household Size and Population Growth Rate is 5.1 and 1.6 respectively. The district has 1 government hospital, 1 non-for-profit hospital, a health center IV, eight (8) HC IIIs and 13 HC IIs.

\subsection{BFHI Implementation and Design}

The study was done as part of BFHI implementation in 24 health facilities however only 12 health facilities qualified for external assessment. The road to baby friendliness as recommended by the $\mathrm{MoH}$ was followed as shown in Figure 1 .

\subsubsection{BFHI Baseline Assessment}

BFHI started with a baseline assessment that sought to understand the health system, 


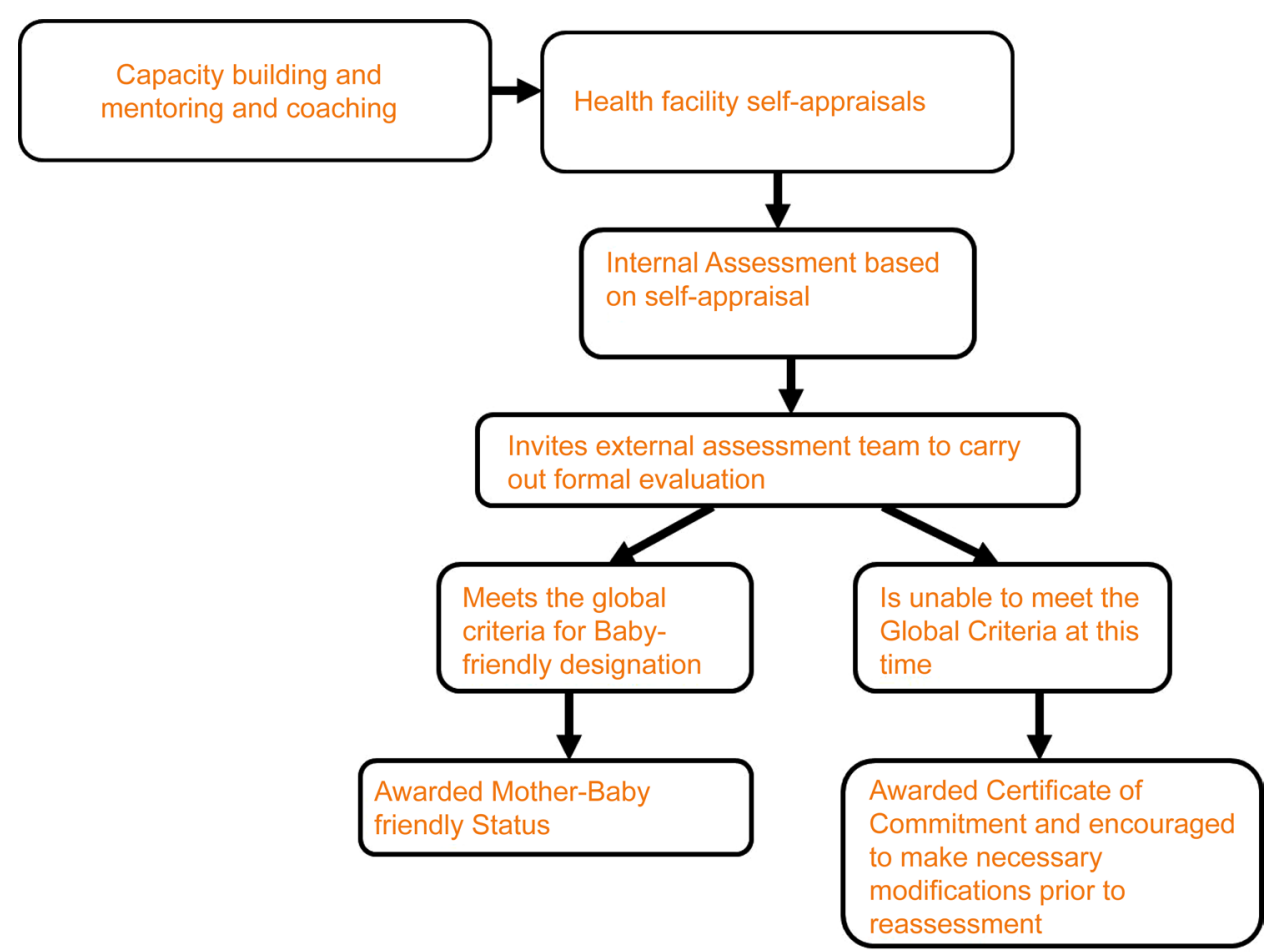

Figure 1. Route to mother-baby friendly accreditation for Kitgum district health facilities [8].

the quality of services and capacity of the health workers to implement BFHI and the linkages and opportunities that implementation of BFHI brings to the achievement of results related to Integrated management of childhood illnesses (IMCI), Prevention of mother to child transition of HIV (PMTCT) and the Ugandan Essential Maternal and Neonatal Clinical (EMNoC) Care. Ministry of health (MoH) BFHI tools were used for this assessment. It targeted the district health team for system strengthening, health workers and the health facility supplies and logistics. This was mainly to understand the district plans and practices to support health facilities to provide quality maternal and child health services. This targeted district hospitals, health center (HC) IV, IIIs and IIs. For the Health centers 11 the $\mathrm{MoH}$ tool was abridged to take care of the $\mathrm{MNCH}$ services offered at that lower level.

\subsubsection{Capacity Building for Health Workers}

The baseline study indicated limited knowledge and skills among health workers to implement BFHI. Against this World Vision Uganda supported capacity building of healthcare providers on BFHI as a starting point to transforming health facilities into mother-baby friendly centers. The capacity building process followed a cascade from district ToTs who then rolled out to staff from key MNCH contact points in the health facilities. The training program was in such a way that all health workers get trained.

\subsubsection{BFHI Mentorship and Coaching}

After the training sessions were completed, the main emphasis was put on making sure that transformation takes place in the Health facilities. For this to happen the health fa- 
cilities were visited periodically by the DHT together with a senior mentor certified as a trainer by the $\mathrm{MoH}$. The visitations aimed at mentoring the Health facility staff on the key specific gaps identified during the baseline and the periodic self-appraisals focusing on the key service provision contact points in the health facility.

The HCs were encouraged to select a BFHI focal person and a team that would then follow up on the key actions agreed upon in case of gaps. However the key skills required were counter demonstrated immediately during the mentoring sessions. The process always engaged the DHT for briefing, seeking permission in addition to following up the issue that could require the intervention of the leadership.

The DHO assigned one DHT member to be the BFHI focal person who spearheads the mentorship process. This strategic direction was meant to increase ownership of the program and strengthening the district capacity to conduct mentoring and coaching. The feedback process started with a Continuous Profession Development (CPD) session on BFHI to all health staffs, followed by joint development of an action plan for improvement or maintenance based on the findings from the exercise.

\subsubsection{Self Appraisal, Follow up and Mentorship}

As part of the transformation process, the HCs are required to keep on conducting periodic self-assessments to realistically gauge their performance. The BFHI focal person at the $\mathrm{HC}$ leads this process using the self-assessment tool focusing on the key $\mathrm{MNCH}$ contact points and leads the assignment of roles to department heads for improvement. Interactive sessions and health facility visits were conducted by the district BFHI focal persons to support health facilities to conduct self-appraisals. During these visits, health workers (HWs) that participated in the self-assessment received technical support on how to achieve the various BFHI health facility requirements. HWs were also continuously and regularly followed up through phone calls. This was mainly to establish extent the HCs are implementing the action plans developed during the mentoring and coaching.

\subsubsection{Learning Event for BFHI Coordinators}

After progressive implementation of BFHI through mentorships and on-site support, success was registered although not at the same rate. This therefore called for organizing learning evets with a purpose of HCs learning from each other as well as provide feedback on the BFHI progress. The Learning events were BFHI step linked, where HCs that performed well shared experiences on how they have managed while the ones with gaps in a particular step picked one or two encouragements from the other. The periodic two day learning events provided a platform for HCs to share experiences with BFHI implementation and also re-oriented them in areas that proved to be technically challenging.

\subsubsection{Internal Assessment}

After registering success through mentorships and on-site support, there was need to prepare the HCs for accreditation. According to the MoH Uganda guidance, for this to 
happen the $\mathrm{MoH}$ needs to invite the independent external assessors but for this to happen the $\mathrm{MoH}$ needs to prepare the facilities. The External assessors do not assess HCs that are not ready for accreditation. For the $\mathrm{MoH}$ to ascertain which $\mathrm{HC}$ are ready for accreditation an internal assessment is done using the tools that the HCs were using for self-appraisal and for mentorship.

This assessment of HCs enabled the $\mathrm{MoH}$ to determine the success of BFHI implementation in the HCs and established the gaps that require to be addressed before accrediting these health facilities as mother-baby friendly. The assessment was conducted in all HCs and those that had progressively improved in BFHI implementation and scored above $75 \%$ were considered for external assessment. The teams spent one day in the hospital and half day in the lower level health facilities.

\subsubsection{External Assessment}

The MoH liaised with The International Baby Food Action Network (IBFAN) Uganda to identified 6 BFHI experts who conducted the external assessment. The BFHI external assessors employed a three stage process i.e.

i) Orientation was conducted for the external assessors using the standard BFHI external assessment tool obtained from UNICEF.

ii) Filed visits to the individual health facilities to collect data; and

iii) Data aggregation and report writing.

The external assessors worked in teams of three at the hospitals and in teams of two for the lower level health facilities. In each team, a lead assessor guided the entire process, including: guidance on selection of the required samples, ensuring the timing and quality of data collection, tallying and scoring of the results.

\subsection{Data Management Processes for This Paper}

The whole process of transforming the 24 HCs to become Mother-baby Friendly took three years of implementation. As stated earlier this process began with a thorough baseline, periodic mentorships and self-appraisals, external assessment and internal assessment. All these processes were data intensive and collection of data was being done at every stage. At the commissioning of the whole transformation process, the project included a strong Monitoring and Evaluation process, in order to document, store and utilse this information effectively.

The tools that were used during this whole process were $\mathrm{MoH}$ approved standard tools and therefore came with already developed data entry templates and were meant to generate simple percentage statistics that eventually lead to the scores required for a health facility to be mother-baby Friendly. For purposes of this publication, the data utilised is an outcome of the process flow for BFHI. During implementation data was carefully processed and stored for further use.

\subsection{Ethical Considerations}

The $\mathrm{MoH}$ approved and supervised the whole transformational process as a contribut- 
ing to quality assurance of the National Health Service programme. The methodology and tools are of the internationally agreed standards. In addition, as part of the end of project for the EAMNCH protocol, the process was approved by the Makerere University school of public health internal review board and further approved by the Uganda national council for science and Technology (UNCST) under reference number SS 5029. All participating health facilities had expressed their commitment to BFHI and the associated assessment procedures. Staff members and women/mothers were interviewed only after verbal consent and confidentiality was maintained throughout the assessment.

\section{BFHI Implementation Results}

\subsection{Demographics}

The plan for the BFHI implementation was meant to support all the 24 HCs in Kitgum District in accordance to the Development plan of the district [9] since it's a health system's strengthening approach targeting the entire health care continuum of care. However the inclusion criteria into this study was based on the fact that the facilities needed to have participated in either the baseline or internal assessment as this qualified the HCs for external assessment and therefore accreditation. In this regard 21 health facilities of which majority were HC IIs (10), HC IIIs (08), hospital (02) and HC IV (01) as indicated in Table 2 met the inclusion criteria. The health facilities that did not participate at the baseline stage were either closed or did not offer any $\mathrm{MNCH}$ services and therefore could not meet the criterial for any assessment. After the District orientation that involved in-charges of all the health facilities signing commitment forms to implementing and participating in the BFHI, those who agreed to include $\mathrm{MNCH}$ services in their routine services were then included in the rest of the activities aimed at their transformation.

\subsection{Health Facility Performance and Transformation Results}

The expected results from the BFHI support was to transform the Health facilities into provision of the services focusing on making sure the BFHI set standards are met. As the HWs performed given MNCH services the HCs were assessed basing on those services in comparision to the required 16 BFHI steps. Each of the step has detailed practices which are cumulatively presented as a percentage for that particular HC. At all levels of the implementation health facilities were assessed to ascertain if the Global BFHI standard [10] is reached. If a health facility has been scheduled for the BFHI assessment and at the time of the assessment the facility was closed its rated Not assessed (NA), any facility scoring between zero to $34 \%$ is not rated, any facility between $35 \%$ and $49 \%$ is Certified to have participated in the BFHI process (CP), that between $50 \%$ and $74 \%$ is Certified Committed to BFHI (CC), and lastly any facility above $75 \%$ is considered Certified Baby Friendly. However a facility only attains the BFHI status after the $\mathrm{MoH}$ invites independent assessors to confirm the status. 
Table 2. Kitgum district health facilities overall BFHI progress performance over a period of three years.

\begin{tabular}{|c|c|c|c|c|c|}
\hline \multirow[b]{2}{*}{ No } & \multirow[b]{2}{*}{ Health facility } & \multicolumn{3}{|c|}{ Percentage Scores } & \multirow[b]{2}{*}{$\begin{array}{l}\text { BFHI } \\
\text { status }\end{array}$} \\
\hline & & Baseline & $\begin{array}{c}\text { Internal } \\
\text { assessment }\end{array}$ & $\begin{array}{c}\text { External } \\
\text { assessment }\end{array}$ & \\
\hline 1 & KITGUM GENERAL HOSPITAL & 60.4 & 98 & 69 & $\mathrm{CC}$ \\
\hline 2 & ST. JOSEPHS HOSPITAL & 62.2 & 94 & 81 & $\mathrm{CBF}$ \\
\hline 3 & NAMKORA HEALTH CENTRE IV & 40.2 & 74 & & \\
\hline 4 & PAJIMO HEALTH CENTRE III & 40.2 & 64 & & \\
\hline 5 & KITGUM MATIDI HEALTH CENTRE III & 59.3 & 94 & 50 & $\mathrm{CC}$ \\
\hline 6 & OKIDI HEALTH CENTRE III & 52.3 & 81 & 38 & $\mathrm{CP}$ \\
\hline 7 & MUCWINI HEALTH CENTRE III & 55.4 & 75 & & \\
\hline 8 & AKUNA LABER HEALTH CENTRE III & 53.9 & 92 & 40 & $\mathrm{CP}$ \\
\hline 9 & OMIYA NYIMA HEALTH CENTRE III & 68 & 90 & 63 & $\mathrm{CC}$ \\
\hline 10 & LOBOROM HEALTH CENTRE III & NA & 90 & 75 & $\mathrm{CBF}$ \\
\hline 11 & OROM HEALTH CENTRE II & 47.7 & 94 & 75 & $\mathrm{CBF}$ \\
\hline 12 & OBYEN HEALTH CENTRE II & 38.3 & NA & & \\
\hline 13 & PUDO HEALTH CENTRE II & 61.4 & 67 & & \\
\hline 14 & GWENGCOO HEALTH CENTRE II & 59.3 & NA & & \\
\hline 15 & AKILOK HEALTH CENTRE III & 57.9 & 92 & 75 & $\mathrm{CBF}$ \\
\hline 16 & LUKWOR HEALTH CENTRE II & 49.5 & 92 & 57 & $\mathrm{CC}$ \\
\hline 17 & LAGOT HEALTH CENTRE II & 57.1 & 87 & NA & \\
\hline 18 & KITGUM TOWN COUNCIL HEALTH CENTRE II & 36 & 52 & & \\
\hline 19 & ORYANGKULUKWA HEALTH CENTRE II & 52.4 & 95 & 40 & $\mathrm{CP}$ \\
\hline 20 & DIOCESE OF KITGHUM HEALTH CENTRE II & 36.7 & 94 & 64 & $\mathrm{CC}$ \\
\hline 21 & NEWLIFE MEDICAL CENTER II & 37.7 & 36 & & \\
\hline 22 & PAJIMO MILITARY HEALTH CENTRE II & NA & NA & & \\
\hline 23 & PAWIDI HEALTH CENTRE II & NA & NA & & \\
\hline 24 & BREGMA MEDICAL CENTRE II & NA & NA & & \\
\hline
\end{tabular}

$\mathrm{NA}=$ not assessed, $\mathrm{CC}=$ certified committed to $\mathrm{BFHI}(50 \%-74 \%), \mathrm{CBF}=$ certified baby, friendly $(75 \%$ or more), $\mathrm{CP}=$ certified to have participated in the BFHI process $(35 \%-49 \%)$.

\subsubsection{Baseline Processes and Key Results}

The baseline sought to understand the quality of services and capacity of the health workers to implement BFHI as a critical component in strengthening $\mathrm{MNCH}$ services. Forty eight (48) HWs were interviewed in 20 HCs followed by a Desk reviews of the existing documents on BFHI. Further focus was placed on the DHT for district plans to support HCs provide MCHN services with a bias in the BFHI. As seen in Table 2 above, the best facility Omiya nyima Health centre scored $68 \%$ while the worst Kitgum town council scored $36 \%$. This simply means that at the onset of the project, there was 
no HC in Kitgum that was mother-baby friendly. Most of the HCs (12) were rated as committed to BFHI, 8 were rated as having participated in the BFHI exercise while the remaining 4 were rated as not assessed. Among identified gaps were, Lack of knowledge and skills required for implementation of BFHI, weak community linkages and lack of visible information materials on $\mathrm{BFHI}$ as required by the criteria.

With the above performance, using a health system strengthening approach, there was need to build the capacity of HWs to integrate the different components of service provision at the contact points using the BFHI framework through training, regular mentoring and coaching. Advocating to the district to develop a mentoring and coaching plan for the HCs rather than check spot supervisions was also key in improving the quality of services.

Supporting the district and HCs to develop and understand the BFHI policy and establish mechanisms to evaluate it, as well as equipping them with equipment needed for the transformation.

\subsubsection{Establishment of BFHI Implementation Structure and Process}

The global criteria focuses on health facilities that have a fully operation $\mathrm{MNCH}$ services provision including the offering of delivery and blood transfusion. However the Ugandan criteria adapted the tools and assessment criteria to include level iii health facilities. These offer both ANC, Young child clinic and maternity services but without conducting caesarian section. However in the system's strengthening approached applied in this project area, the assessment and implementation criteria was further adapted to include the HC ii that only offer ANC, Young Child Services and conduct emergency deliveries. This was because the Kitgum district is majorly covered by 13 HCs at this level as seen in Table 2. The criteria adaptation was speared headed by the $\mathrm{MoH}$ in partnership with the District Health team.

The Process was then followed by the following key processes below:

i) Orientation of the leadership authorities to offer commitment to the implementation of BFHI

In an effort to ensure a close working relationship and ownership of the BFHI programme at all levels, an orientation meeting for the Kitgum district leadership was held before the activities were rolled out. As such, orientation of the DHT, MNCH/Nutrition stakeholders and all in-charges of the HCs on the BFHI implementation was earmarked as a way of promoting system strengthening, and to ensure that the district identifies key officers to monitor the implementation closely. The district leadership and all health centre in-charges committed to supporting the transformation processes and to avail service providers for mentorship.

Key on the agenda of this meeting was to set a platform for implementation of BFHI through discussions with the district leadership and planning together to achieve the objectives, share with the district the developed and planned activities that will be implemented in ensuring that quality of service delivery improves using the BFHI strategy, and disseminate findings from the BHFI baseline assessment of the HCs. 
The leaders were able to understand the BFHI implementation plan and highly appreciated the initiative that was aimed at contributing to reduction of maternal and child morbidity and mortality in the district. The district leadership therefore pledged commitment to support the implementation of BFHI, beginning with transforming their work place into a baby friendly that supports breastfeeding at all times. The MNCH Focal person and the District Nutritionist were appointed focal persons to coordinate and support the implementation of BFHI. Further still all health facilities that participated agreed to establish BFHI implementation structures including having a BFHI coordinator and monitoring committees.

\section{ii) Training of frontline service providers in BFHI}

The initial process involved training 69 health workers from $23 \mathrm{HCs}$, only one did not send any participant to the training. The package used was the $\mathrm{MoH}$ approved BFHI training curriculum. The training was spread along 3 weeks starting with the in-charges of the HCs and then the front line staff who work at the ANC clinic, the Maternity, post-natal care units, the Young Child Clinic and the HIV/AIDS care clinic.

During the training the participants were enlightened on how to appreciate the role of BFHI in improving quality of service and its linkages with EMNoC and IMCI, equip HWs with knowledge and skills to support mother to successfully breastfeeding their children, update health workers on current IYCF recommendations from the $\mathrm{MoH}$ and identification of potential BFHI coordinators for each health facility and at the district level.

As part of the capacity building blocks, Information Education and Communication (IEC) materials were also distributed to all HCs and these facilities were supported to development their own improvement plans and BFHI policies with the guidance of the National BFHI steps. The HCs also needed to identify the Baby friendly corners and demonstration materials were provided to facilitate continuous health education to mothers at the contact points.

\section{iii) Health facility self-appraisal and periodic mentorships}

Following the capacity building activities the HWs were given sometime to go back to the HCs and to implement the actions. Top on the agenda for this was to self-generate their own BFHI policy in the local language and then set up the BFHI implementation framework basing on the gaps identified during the baseline process. This was followed by the initial mentorship where senior BFHI trainers worked with the HC staff to understand the self-appraisal tool, interpret the results of each BFHI step and what actually each step requires in terms of the actual key $\mathrm{MNCH}$ contact point implementation.

The outcome of this was that HWs on a monthly basis were able to assess their facilities performance in terms of BFHI implementation. The HCs conducted the self-appraisals using the BFHI tool. They were able to identify their weaknesses or gaps and develop actions to address them. Through the regular self-appraisals, HCs were able to improve performance and indicate if they qualify for an internal assessment. HCs were followed up to ensure they conduct the self-appraisals spearheaded by the BFHI District 
coordinator and a staff who was recruited as a secondment to the District by the EAMNCH project.

\section{iv) Preparation of the Health facilities for the Internal assessment}

The preparation of HCs for the internal assessment involved one key mentorship with a focus on the services provided to fulfill the requirements of the BFHI steps. And a learning session to facilitate learning from each other in preparation for a major $\mathrm{MoH}$ assessment.

The Learning session agenda emphasized a number of practices that would be useful in improving the implementation of particular BFHI steps and this would in turn contribute to the overall score of the $\mathrm{HC}$ and therefore prepare it for accreditation as mother-baby friendly. The HCs needed to exchange successes and failure on having a policy displayed in a language that is easily understood by its clients and HWs regularly communicate the policy to its clients, prioritize and document BFHI issues discussed during Monthly continuous medical education sessions (CMEs), document all health education topics discussed with clients and ensure that the schedule is displaced in areas where Health education is given, place all new born babies in skin to skin position and supported to breast feed within one hour of life and inform mothers of the importance of all BFHI practices and be encouraged to continue lactating until 2 years.

The focus was also placed on reinforce the existing community structures while empowering them with information and motivating them so that they can support mothers in the community, HCs issuing out an appropriately fully filled mother-child passports and mothers are always encouraged to attend PNC and the young child clinics for follow up. The emphasis was placed on these key issues because they came out as molar skills requirements that will determine how the health facilities will be rated by the $\mathrm{MOH}$ during the internal assessment. Health workers in these facilities needed to demonstrate that they practice them on a day to day basis not through their knowledge but through their skill set.

\subsubsection{Health Facility BFHI Internal Assessment Performance in Kitgum District}

All continuously supporting the HCs, it was clear that only $21 \mathrm{HCs}$ of the 24 were actually ready for a BFHI internal assessment. It was against this background that Kitgum district requested the $\mathrm{MoH}$ to internally assess the $21 \mathrm{HCs}$ in order to ascertain their readiness external assessment.

The results from the internal assessment determine which HCs are included in the external assessment. As presented in Table 2, only 5 HCs were able to score $91 \%$ - 99\% on the assessment tool and therefore qualified directly for external assessment. These included Kitgum General Hospital, two Health centre iii and two health centre ii.

Nine of the health centres scored between $75 \%$ - $90 \%$ on the facility assessment tool and therefore showed commitment to BFHI. This therefore called for another set of mentorships to be able to have these facilities work on the gaps. These include St. Joseph Hospital, five Health centre iii and three Health centre ii. A re-assessment of these facilities was done and from the $9 \mathrm{HCs}$ only 7 managed to attain a readiness status, the 
other 2 could not qualify.

Five health centres scored between $36 \%-74 \%$ on the requirement tool and were automatically dropped from further assessments. This is simply because they needed further mentorship, self-appraisal sessions to be able to catch up. These included Namukoro Health centre iv, two health centre iii \& ii. However the number of those that did not participate in the external assessment increase to 7 after the re-assessment as seen in Table 2.

It should be noted that of the 21 health facilities planned for the internal assessment two were not assessed. The reason sited here was because the facilities did not avail service providers to be assessed at the time of the scheduled visit. The facilities were actually closed.

\subsubsection{BFHI External Assessment Performance}

This was mentioned as the forth [11] BFHI external assessment conducted by the $\mathrm{MoH}$ since the adaptation of the Innocenti declaration in Uganda. It involved the 12 health facilities of which 2 were hospitals, 6 HCiii and 4 HCii. From the assessment and basing on the global set standards, Four (4) out of twelve (12) HCs scored $75 \%$ and above, and this is the required minimum score for a health facility to be certified as Baby Friendly. The four are: St Joseph's Hospital (81\%), Orom HCiii (75\%), Loborom HCiii (75\%), and Akilok HCiii (75\%). All the four HCs actually offer fully fledged ANC, Maternity and Yung Child clinic services but only St. Joseph's hospital offers C-section, incubation for babies born too soon and low birthweight babies and blood transfusion.

As seen from Table 2: Five (5) of the 12 Health facilities assessed attained a Certified Committed to BFHI status with scores ranging from $50 \%$ to $74 \%$. These included Kitgum general hospital, Omiya nyima HCiii, Kitgum matidi HCiii, Diocese of Kitgum HCii and Lukwor HCii. Three (3) of these health facilities offer ANC maternity and Young child clinic services. Only two of these offer ANC and Child clinic services with offering only emergency deliveries if they occur in the geographical coverage. Only Kitgum general hospital offers C-section services, incubation for babies born too soon, low birthweight babies and blood transfusion. It should be noted that Kitgum general hospital is the districts main referral hospital.

Three (3) of the 12 health facilities assessed attained a Certified to have participated in the BFHI process with a score between $35 \%$ to $49 \%$. These included Okidi HCiii, Oryang Kulkwa HCii, and Lukwor HCii. All the three offer ANC and Young child clinic services however only Okidi offers maternity services under normal circumstances.

\section{1) BFHI transformational results for Kitgum}

One of the main objectives of the BFHI project in Kitgum was to transform health facilities to become mother-baby friendly. Right from the baseline through the mentorships and self-appraisal processes, the project team used the same tool to assess functionality of BFHI. Focusing on the $12 \mathrm{HCs}$ that went beyond the internal assessment, the project decided to run am improvement score analysis for the 12 health facilities to ascertain the greatest contribution. 
As seen from Figure 2 below on average the district scored a $34.6 \%$ improvement on the MoH Uganda BFHI tool from the baseline to the internal assessment. The highest improvement score was noted by Diocese of Kitgum HCii with an improvement score of $57.3 \%$ noted from a baseline score of $36.7 \%$ to an internal assessment score of $94.0 \%$.

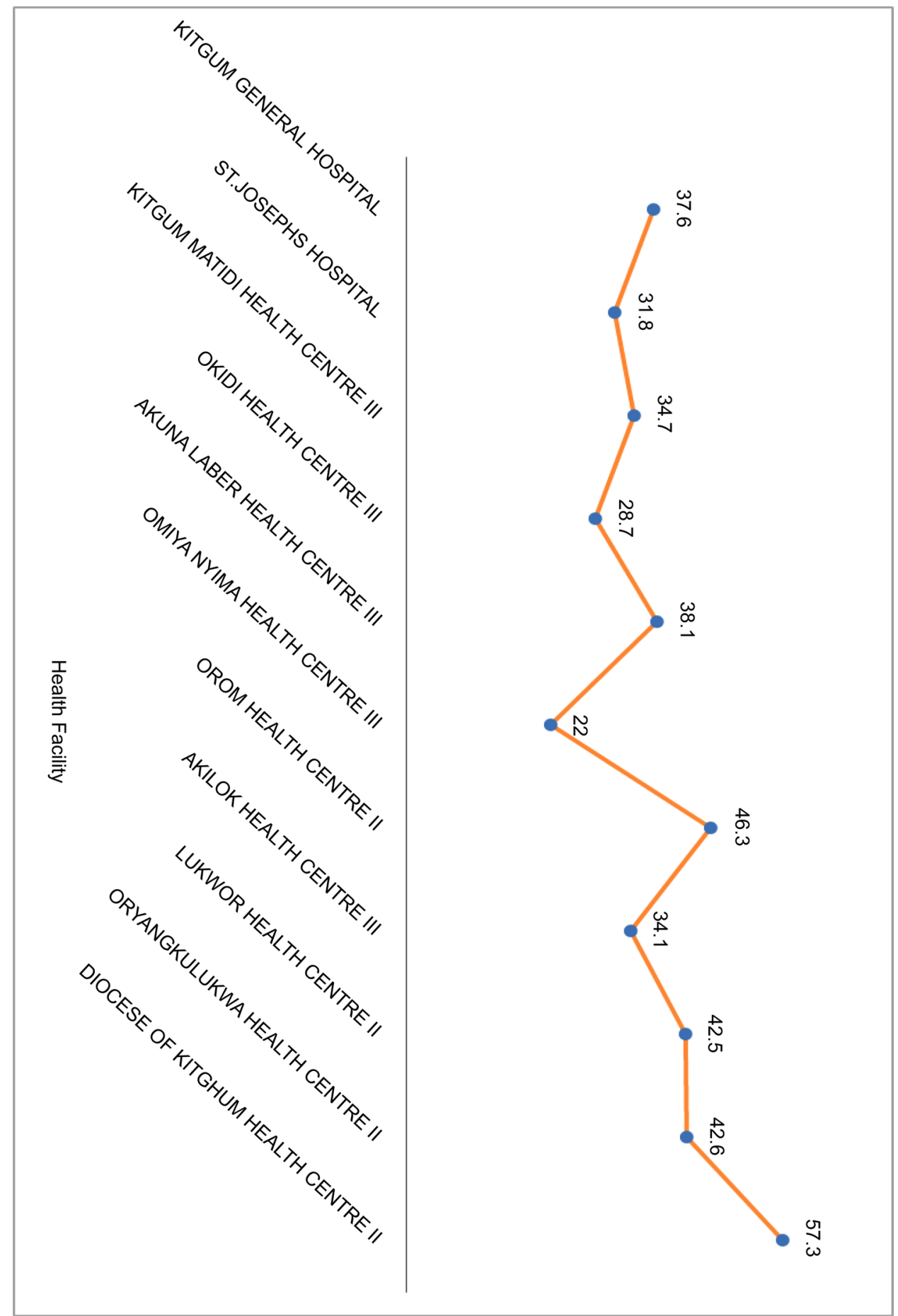

Figure 2. Shows percentage transformation improvement of the 12 health centres from baseline to international assessment. 
The lowest improvement score on the tool was $22.0 \%$ scored by Omiya nyima HCiii noted from a baseline score of $68.0 \%$ to $90.0 \%$ at the time of the internal assessment.

\subsection{MoH BFHI 16 Steps Performance}

\subsubsection{Overall Improvement Performing Step}

From Figure 3 as shown below, Step 1. Have a written breastfeeding policy that is routinely communicated to all health care staff indicated the overall best improvement step. At the baseline, there was no health facility in Kitgum that had a written policy, in the local language that was communicated and understood by all staff and clients that visit the health facilities. After the capacity building and commitment processes, the status improved from zero to $60 \%$ and by the internal assessment period $82.9 \%$ of all HCs had full filled this requirement. It's important to note that for the BFHI implementation to make sense, all HCs being supported must meet this requirement. It's the entry point to any transformation. Without this written policy, communicated to all staff and posted where the clients can actually read and understand it there would be no transformation since it all begins from this policy. The Infant and Young child feeding policy guidelines for 2009 [5] provides the guidance on this and HCs were supported to interpret this for their local context.

\subsubsection{Overall Best Performing Step}

As seen in Figure 3, the step that performed best was Step 8. Encourage breastfeeding on demand. Without even starting the implementation of, HCs were already encouraging mothers who to breastfeed their babies on demand immediately after delivery and when the mothers are discharged. At the baseline this step overall scored $80.95 \%$, after the capacity building processes the score was $100 \%$ and it never changed even at internal assessment remained at $100 \%$. The MoH Infant and Young child feeding policy encourages and recommends breast feeding to be a norm. This step's performance also presents an indication that Kitgum district health care workers believe in the practice of breastfeeding and therefore would not encourage the use of breast milk substitutes, teats or pacifiers to mothers who have just delivered newborns.

\subsubsection{Static Performing Step}

As seen in Figure 3, there were two BFHI steps that had approximately a baseline score similar to the internal assessment score. Firstly was Step 6. Give newborn infants no food or drink other than breast milk, unless medically indicated. At the baseline this score on average was $58.10 \%$, which improved to $69.61 \%$ during the mentorship process but then at internal assessment reduced to $59.26 \%$. This implies that there is still cultural practices of giving newborn babies prelacteals feeds after delivery. The other was Step 7. Practice rooming-in-allow mothers and infants to remain together-24

hours a day. The score at baseline was $76.19 \%$ and it rose to $88.24 \%$ at the Mentorship and reduced to $77.08 \%$. The average percentage is not that alarming, however this should not downpour the value of mothers rooming or bedding in with their newborns as this promotes bonding and facilitates breastmilk letdown. 


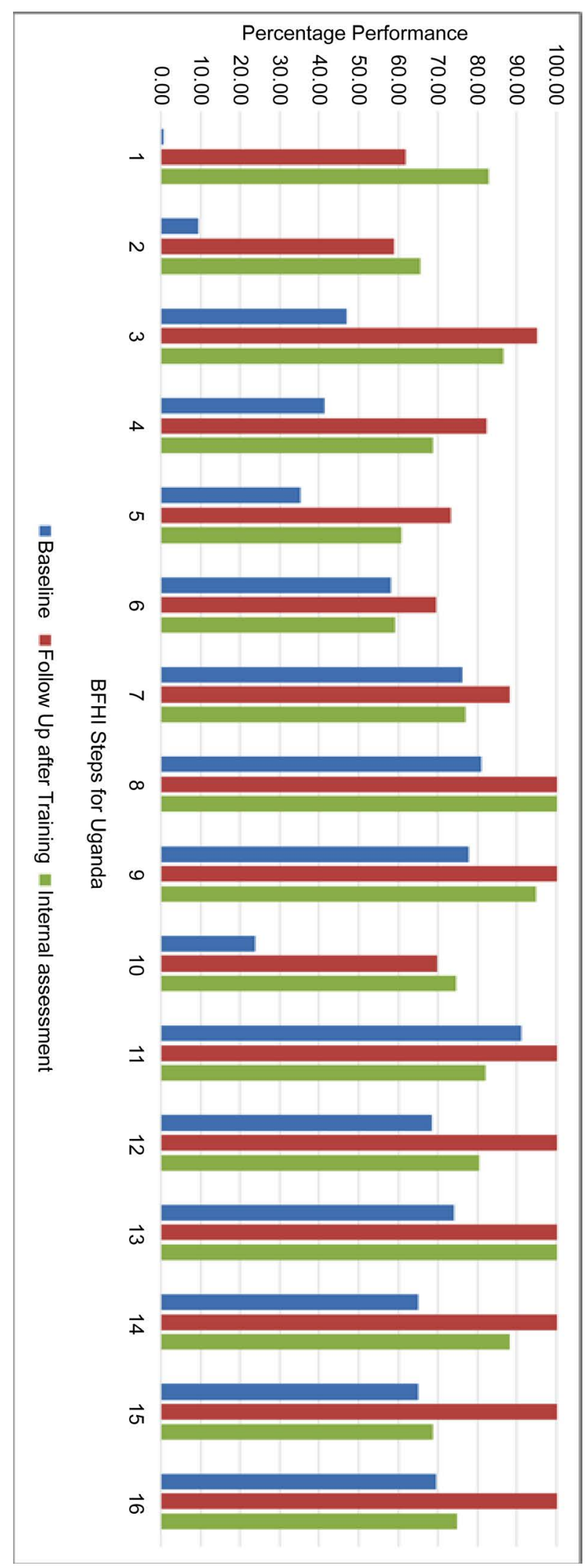

Figure 3. General improvement performance of the BFHI 16 steps for Kitgum. 


\section{Lessons Learnt and Challenges Faced during Implementation}

\section{Lessons Learnt during the Implementation of BFHI in Kitgum District}

1) Focused efforts and support from all stakeholders at the district level in the commitment process to the transformation of HCs for BFHI is crucial in the roadmap to attain the mother-baby friendly status.

2) Periodic collaborative learning sessions conducted for HWs to share experiences and challenges promotes learning and motivates them to perform better and be able to provide mother-baby friendly services.

3) BFHI transformation processes do not require extra HWs or resources within the facilities. The attitude of the HWs is key for the achievement of the mother-baby friendly status and this must be addressed right from training.

4) Reinforcement of the existing community structures while empowering them with information and motivating them to support mothers in the community is important ensuring continuity of the BFHI practices at home.

5) Routine Continuous Medical Education held at all health facilities and documented regularly should include BFHI requirements as this enables better understanding of BFHI and how it can be integrated in routine health services.

\section{Transformational Challenges during Implementation}

i) Weak community linkages as majority of the facilities have not established the mother support groups. This is a requirement for the continuity of support and practice at the household level.

ii) The health facilities that had mother support groups operational were not able to integrate infant feeding aspects since the transformation process had a lot of deliverables in a limited time.

iii) The high staff attrition affected the technical capacity of the HCs to implement BFHI since some staff that were trained either transferred or found other opportunities and this made the process more of a capacity building process with limited actual transformational implementation timelines.

\section{Discussion, Conclusion and Recommendations}

\subsection{Discussion}

It remains to be noted that a number of challenges ranging from financial to logistical have slowed the transformation process for BFHI as this could be noted from the low number of accredited facilities in Uganda so far. It was against this background that the $\mathrm{MoH}$ and partners continue to promote BFHI efforts in ensuring that at least the number of HCs designated baby friendly increase from fifteen to seventy as stipulated in the Health Sector Strategic and Investment Plan [12]. In Uganda where only 57\% of the deliveries take place in HCs, it is important to give initial emphasis on improving facility level essential and emergency care for mothers and newborns; using the Ugandan Essential Maternal and Neonatal Clinical (EMNoC) Care including the emergency Obste- 
tric Care (EmOC) and expanding the Integrated Management of Newborn and Childhood Illnesses (IMCI) to include neonates strategies. At the same time, forging linkages with communities, especially for birth planning, health education and post-partum care through the famous Ugandan Village Health Team (VHT) that has proved vital in the health care service delivery.

The success of BFHI therefore depends on upgrading of HCs to provide EMNoC which focuses on goal oriented ANC, EmOC, labor management, competency based training on life saving skills and maternal death review. Since the focus has mainly been on mothers, IMCI protocol needs to re-emphasize so that children are cared for and such illnesses prevented, hence the success of BFHI since its overall goal is for facilities to provide services that address the health of the child and the mother so that they are both healthy without compromising breastfeeding. The capacity of health workers should therefore be expanded to link the interventions promoted in the Ugandan EMNoC so that all mothers attend the four recommended visits, deliver in HCs with a skilled HWs among others and then early management of common childhood illnesses like pneumonia, diarrhea and Acute respiratory infections. This successfully contributes to the requirements of BFHI and therefore provision of mother-baby friendly services.

The transformation of health facilities into mother-baby friendly utilised a behavioral change approach targeting the HWs since most of the requirements for BFHI are the routine services they offer or that they are usually reluctant to provide. The implementation therefore based on self-realization of the gaps and finding solutions to addressing them based within a particular health facility.

Since breastfeeding is an essential component of newborn survival, supporting BFHI and maintaining its principles is key in improving maternal and child health. Besides linking with EMNoC interventions to ensure all facilities are baby-friendly, it is recommended that requirement 10 of the 16 steps of BFHI, the community component, be strengthened and solutions to provide support for women in communities be designed based on local realities (Figure 4).

The uniqueness of BFHI is that it is a step wise approach which the health workers should be aware of and are willing to implement since these are the routine activities that HWs conduct.

For example, requirements 3 promotes goal oriented Antenatal care and requirement 12 is about delivery services and ensuring good birth outcomes. Requirement 13 of BFHI shows the linkage with PMTCT, as it assess the feeding options and competencies of health workers to support HIV positive mothers to feed their babies.

BFHI approach prepares the mother right from during pregnancy until she gives birth by strengthening the implementation of EMNoC through urging health workers to provide goal oriented ANC, preparing the mothers for labor and delivery and ensuring they are healthy throughout by providing the key medications and supplementation like Vitamin A during postpartum care. The linkage between BFHI and EMNoC is strong and once health workers understand it, they can fulfill the requirements and easily 


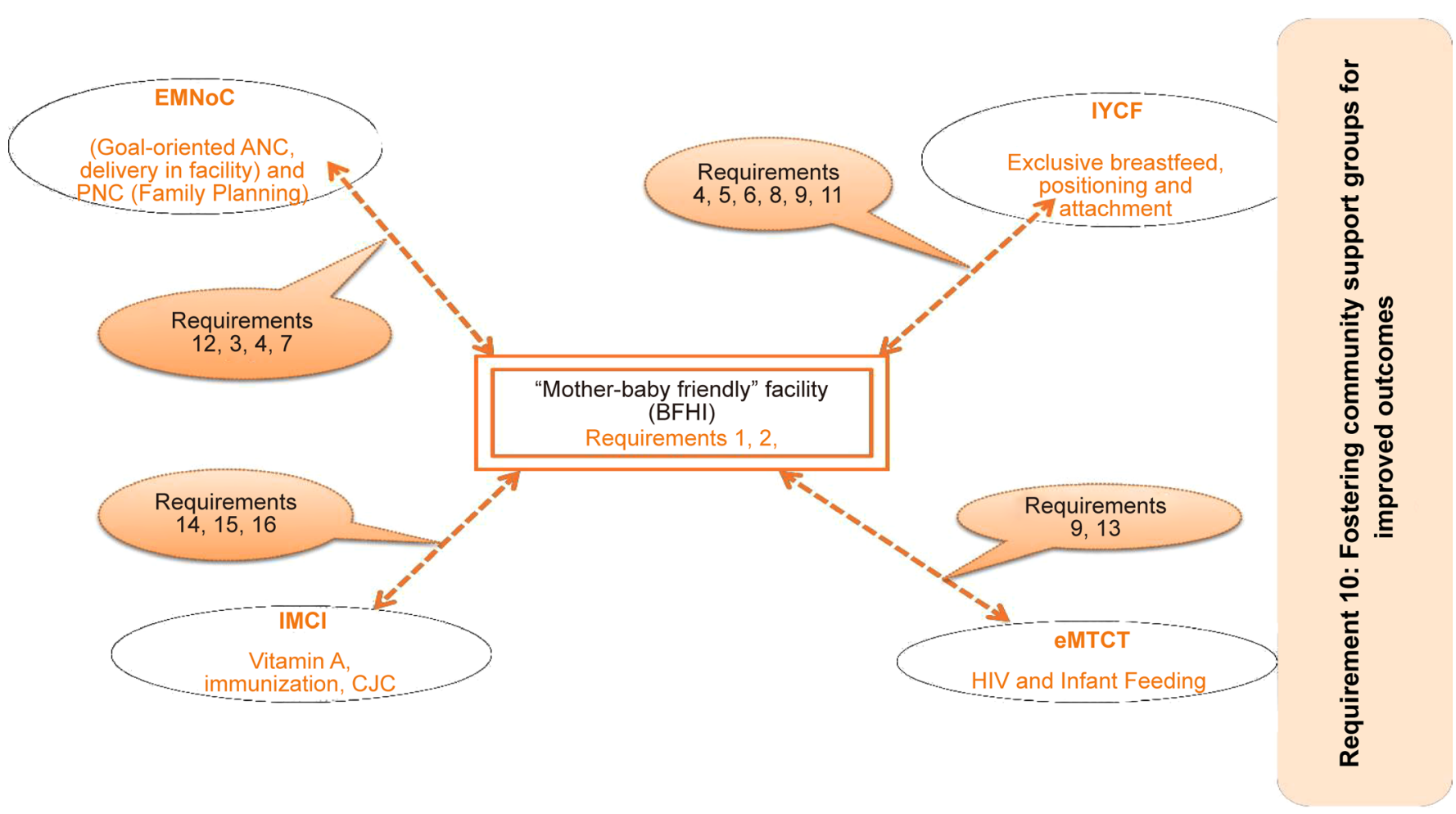

Figure 4. Linkage of IMCI, EMNoC, IYCF and PMTCT to BFHI [13].

be designated mother-baby friendly facilities as they are able to support mothers to breastfeed, and also remain healthy. Figure 4 demonstrates the intended achievement and linkages at health facility skills practice level on what packages were being scored when the implementation of BFHI was taking place. Through the support of health centres to implement BFHI they were able to achieve the integration of key $\mathrm{MNCH}$ packages through one implementation approach.

\subsection{Conclusion}

Four (4) out of 24 HCs in Kitgum district were able to attain a Baby-Friendly status following a comprehensive transformational process towards excellence in providing evidence-based maternity and newborn care. The goal was to achieve optimal infant feeding outcomes and mother-baby bonding. The process for all the health facilities that got involved was exciting, challenging, and worth to venture due to numerous benefits. Benefits of the BFHI program included creating opportunities to develop high performance work teams, building leadership skills among staff and promoting employee satisfaction. Further, it enhanced patient satisfaction and improved maternal and newborn health outcomes at the health facility level. The EAMNCH project is applauded for following the due process and strategically following the path of health systems strengthening. Much as the country has embraced the BFHI approach as a means of improving the IYCF practices, implementation has been limited to few areas where there is partner support. Besides the quality of BFHI implementation has been com- 
promised by lack of commitment from the health workers, coupled with weak routine monitoring and supervision.

\subsection{Recommendations}

a) The MoH should incorporate BFHI periodic assessment and mentorship in routine Quality Assurance Programme and advocate for the sensitization of District Local Governments and other stakeholders to support BFHI.

b) The District Health Teams should ensure that there is a BFHI focal person at district level and that this person remains active to encourage health facilities continue with BFHI implementation and provide a basis for resources allocation for the BFHI related activities.

c) Health facilities should intensify routine communication to staff members on the BFHI policy and ensure that the health workers implement practices as per policy. This could be done through continuous medical education sessions.

d) Development and implementing partners like World Vision are requested to increase their technical and financial support for BFHI implementation and assessment to contribute to the geographical coverage of BFHI within the country.

\section{Acknowledgements}

The East African $\mathrm{MNCH}$ project was implemented with support from Australia Africa Community Engagement Scheme, (AACES) funded by the Government of Australia, Department of Foreign Affairs and Trade (DFAT) through World Vision Australia. Funding was utilised by World Vision Uganda in partnership with the Kitgum District Local government and the Diet Clinic Uganda from 2011 to 2016.

\section{References}

[1] WHO/UNICEF Baby-Friendly Hospital Initiative (BFHI) (2003). http://www.breastcrawl.org/breastfeeding-bfhi.shtml

[2] UNICEF (1990) Innocenti Declaration: On the Protection, Promotion and Support of Breastfeeding.

http://www.who.int/about/agenda/health_development/events/innocenti_declaration_1990. pdf

[3] WHO (2013) Implementation of the Baby-Friendly Hospital Initiative Biological, Behavioral and Contextual Rationale. http://www.who.int/elena/bbc/implementation_bfhi/en/

[4] Ministry of Health (2009) Baby Friendly Health Facility Initiative: A Training Course Revised Updated and Expanded for Integrated Care. Uganda.

[5] Ministry of Health (2009) Policy Guidelines on Infant and Young Child Feeding. Uganda.

[6] Ministry of Health (2011) The Baby Friendly Health Facility Initiative, Health Facility Assessment Tool. Uganda.

[7] The Diet Clinic (2013) Baseline Study on Health Facility Capacity to Implement Baby Friendly Health Facility Initiatives in Kitgum District.

[8] The Global Criteria for the BFHI (2009) Baby-Friendly Hospital Initiative: Revised, Updated and Expanded for Integrated Care. 
[9] Republic of Uganda (2012) Kitgum District Local Government Five-Year Development Plan. http://npa.ug/wp-content/themes/npatheme/documents/North/Kitgum\%20DDP.pdf

[10] Baby-Friendly Hospital Initiative (2009) Revised Updated and Expanded for Integrated Care. Section 4: Hospital Self-Appraisal and Monitoring. http://unicef.org/french/nutrition/files/BFHI_2009_s4.pdf

[11] Mukasa Gelasius, IBFAN Uganda and Namukose Samalie Bananuka, Ministry of Health, (2014) Report on the Baby Friendly Health Facility Initiative (BFHI) External Assessment in Kitgum District.

[12] Ministry of Health. Health Sector Strategic and Investment Plan. Uganda.

[13] The Diet Clinic (2011) A Concept Note on Transforming Health Facilities in Kitgum District to Become "Mother-Baby Friendly" Using Baby Friendly Health Facility Initiative (BFHI) Strategy.

Submit or recommend next manuscript to OALib Journal and we will provide best service for you:

- Publication frequency: Monthly

- 9 subject areas of science, technology and medicine

- Fair and rigorous peer-review system

- Fast publication process

- Article promotion in various social networking sites (LinkedIn, Facebook, Twitter, etc.)

- Maximum dissemination of your research work

Submit Your Paper Online: Click Here to Submit

Or Contact service@oalib.com 